\title{
Prevalence of diabetic retinopathy in children and adolescents with IDDM
}

\section{A population-based multicentre study}

\section{A . Kernell ${ }^{1}$, I. D edorsson ${ }^{2}$, B. J ohansson ${ }^{3}$, C. P. Wickström ${ }^{4}$, J . L udvigsson ${ }^{1}$, T. Tuvemo ${ }^{5}$, J. Neiderud ${ }^{6}$, K. Sjöström ${ }^{7}$, K. M almgren ${ }^{8}$, P.K anulf ${ }^{9}$, L. M ellvig ${ }^{10}$, M. G jötterberg ${ }^{11}$, J. Sule ${ }^{12}$, L . A . Persson ${ }^{13}$, L. I. L arsson ${ }^{14}$, J. A $\operatorname{man}^{15}$, G . D ahlquist ${ }^{16}$}

${ }^{1}$ Department of Paediatrics, University Hospital, Linköping, Sweden

${ }^{2}$ Department of Ophthalmology, Örebro Medical Centre Hospital, Örebro, Sweden

${ }^{3}$ Department of Ophthalmology, University Hospital, Linköping, Sweden

${ }^{4}$ Department of Ophthalmology, University Hospital, Uppsala, Sweden

${ }^{5}$ Department of Paediatrics, University Hospital, Uppsala, Sweden

${ }^{6}$ Barnkliniken, Helsingborgs lasarett, Helsingborg, Sweden

${ }^{7}$ Department of Ophthalmology, Helsingborg Hospital, Helsingborg, Sweden

${ }^{8}$ Department of Paediatrics, Ryhov Central Hospital, Jönköping, Sweden

${ }^{9}$ Department of Ophthalmology, Ryhov Central Hospital, Jönköping, Sweden

${ }^{10}$ Department of Paediatrics, Karolinska Institute, Huddinge Hospital, Stockholm, Sweden

${ }^{11}$ Department of Ophthalmology, Karolinska Institute, Stockholm, Sweden

${ }^{12}$ Department of Ophthalmology, Karolinska Institute, Södersjukhuset, Stockholm, Sweden

${ }^{13}$ Department of Paediatrics, University Hospital, Umeå, Sweden

${ }^{14}$ Department of Ophthalmology, University Hospital, Umeå, Sweden

${ }^{15}$ Department of Paediatrics, Örebro Medical Centre Hospital, Örebro, Sweden

${ }^{16}$ Department of Paediatrics, Karolinska Institute, Sachs' Children's Hospital, Stockholm, Sweden

Summary Vision-threatening diabetic retinopathy can be prevented if it is diagnosed before becoming too advanced. Since diabetic retinopathy has been reported to occur only rarely before the end of pubertal development, children and adolescents are seldom included in screening programmes. We invited 780 children and adolescents with insulin-dependent diabetes mellitus diagnosed before the age of 15.0 years (disease duration of $<12$ years) and who were older than 9.0 years at the time of examination from eight regions of Sweden. Retinal examination was performed with stereoscopic fundus photograph. The photograph were rated according to a modified Airlie House classification. The dropouts (223/780, 28.6\%) were significantly older and with a longer duration of diabetes than the examined children $(p<0.001$ and 0.001 , respectively). Photographs from 557 patients aged (median [interquartile range]:14.6 [12.417.0]) years and with a diabetes duration of 8.0 (5.59.9) years were evaluated. Retinopathy was demonstrated in 81 patients (14.5\%):66 with background retinopathy, 2 with microaneurysms and hard exudates, 12 with preproliferative retinopathy, 1 with proliferative retinopathy. Preproliferative retinopathy was diagnosed in a 12.8-year-old girl in pubertal stage 3 and an 11.8-year-old boy in pubertal stage 2, and proliferative retinopathy was found in a 21.5 year-old girl. Retinopathy was demonstrated in $6 \%$ and $18 \%$ of patients in pubertal stages 1 and 5 , respectively. The overall prevalence of retinopathy in this population may even be higher since the dropouts were older and had a longer duration of diabetes. Since background and preproliferative retinopathy were found in children before puberty, we recommend including children and adolescents in screening programmes for diabetic retinopathy from the age of 10 years. [Diabetologia (1997) 40: 307-310]

Keywords Retinopathy, insulin-dependent diabetes mellitus, pubertal development, fundus photography, population-based study.
Received: 18 January 1996 and in revised form: 14 November 1996
Corresponding author: Dr. A. Kernell, Department of Pediatrics, University Hospital, S-581 85 Linköping, Sweden A bbreviations: IDDM, Insulin-dependent diabetes mellitus. 
In most western countries, the leading cause of blindness is diabetic retinopathy, although the prognosis has improved considerably due to advances in laser therapy and vitreoretinal surgery. Laser-photographcoagulation yields the best results if instituted before retinopathy has advanced too far. Screening-programmes have been instituted in several countries [1] aimed at early detection and close monitoring of retinal changes.

When patients should enter such screening programmes has not been completely resolved. Duration of diabetes is invariably reported to be the most important risk factor for retinopathy [2-6]. Almost $100 \%$ of patients with insulin-dependent diabetes mellitus (IDDM) have signs of retinopathy 15 years after diagnosis of diabetes. Diabetic microvascular complications, such as retinopathy, have been reported to be very infrequent before onset of puberty, independent of prepubertal duration of diabetes [26]; thus, it has been suggested that routine examinations would be unnecessary before puberty.

This population-based study was undertaken to determine the prevalence of retinopathy in children and adolescents from the age of 9 years with onset of IDDM before the age of 15.0 years and within 12 years after diagnosis of IDDM in relation to age, duration and pubertal development.

\section{Patients and methods}

In eight different regions in Sweden, children and adolescents with IDDM who were born after 1979 and who had been diagnosed before the age of 15.0 years and between 1 July 1977 and 31 December 1986 were invited to join the study. Since by the time of this study all children with diabetes onset before the age of 15.0 years were referred to a specific paediatric department covering a specific geographic area of residence, this study can be considered truly population based. Using the Swedish childhood diabetes register, which is nationwide and covers all incident diabetic cases since 1 July 1977 with onset before the age of 15 years, it was possible to trace all cases initially recorded by the collaborating paediatric departments. The detection level of this register has been estimated at $99 \%[7,8]$. Children younger than 9 years of age were excluded because of technical difficulties in obtaining a satisfactory fundus photograph. The study period was 1 November 1987 to 1 March 1989.

The 780 patients invited to take part in the study 611 accepted. Photographs from 557 patients were of acceptable quality and could be evaluated. The total number of dropouts were thus 223 of 780 invited patients $(28.6 \%)$. The patients who did not join the study or who had fundus photographs of unacceptable quality were significantly older and had a longer duration of the diabetic disease (Table 1).

The photographs were taken stereoscopically at a camera angle of $45-50^{\circ}$ and covered three fields; optic disc in centre, macula in centre and temporal macula. Three experienced ophthalmologists (I. D., B. J. and C. P. W.) evaluated all the photographs with the aid of the Airlie House standard photographs: Nos. 1, 2B, 3, 4, 5, 6B, 7, 8B, 10A and 10C [9], independently of each other and using a standardised protocol. The grading system was the one used in the KROC study [10]. The
Table 1. Description of the patients with acceptable photographs and of the drop-outs

\begin{tabular}{llll}
\hline & $\begin{array}{l}\text { Acceptable photos } \\
(557)\end{array}$ & $\begin{array}{l}\text { Drop-outs } \\
(223)\end{array}$ & $\mathrm{p}$ \\
\hline $\begin{array}{l}\text { Age (years) } \\
\begin{array}{l}\text { Duration of } \\
\text { diabetes (years) }\end{array}\end{array}$ & $5.4(3.6(12.4-17.0)$ & $18.2(14.1-21.3)$ & $<0.000$ \\
Female/male & $279 / 278$ & $8.0(5.5-9.9)$ & $<0.000$ \\
\hline
\end{tabular}

Subjects were considered to be drop outs when they had nonevaluable photographs or did not attend the study

identity of the photographs was masked to the opthalmologists. The grading was used concomitantly for both eyes.

The protocol, the standard photographs and the grading system were evaluated by nine experienced ophthalmologists (including the three ophthalmologists participating in the present study). They independently graded photographs from 54 patients with different stages of retinopathy. The identity of the photographs were masked to the raters. The inter- and intra-agreement results were evaluated with help of kappa-statistics [11].The kappa value for a photograph evaluated by the same reader twice was 0.98 and for two co-trained raters was 0.90 , i.e. almost perfect agreement. Weighted kappa [12] for total agreement between three raters for microaneurysms and the KROC-grading system was $0.66(z=6.0, p<0.0001)$ and $0.40(z=4.44, p<0.001)$, respectively.

The following clinical parameters were recorded at the time of the eye examination: patients' weight, height, dose of insulin in IU/kg body weight, number of insulin doses per day, pubertal stage according to Tanner (Table 2), testicular volume and age at menarche.

The study was approved by the ethics committee of the Faculty of Health Sciences, Linköping University and all local ethics committees. All patients gave their informed consent.

\section{Statistical analysis}

Results were analysed using JMP software from SAS institute Inc (Cary, N.C., USA). The Wilcoxon signed rank test and Fisher's exact test were used to calculate differences between groups. Life table and Cox proportional hazard analysis were used in order to analyse what factors influenced the survival time without retinopathy [13].

\section{Results}

Signs of retinopathy were demonstrated in 81 of the 557 patients $(14.5 \%$, Table 2$)$. Clinical characteristics of patients with and without any signs of retinopathy are shown in Table 2.

Retinopathy was diagnosed in $5 \%(1 / 19$, Fig. 1$)$ of the youngest patients between 8 and 10 years of age, and in $71 \%(10 / 14)$ of the oldest patients aged between 23 and 25 years. The prevalence of retinopathy increased from $4 \%(2 / 45)$ in patients with less than 2 years' duration of diabetes to $32 \%(9 / 29)$ with a diabetes duration between 10 and 12 years (Fig. 2).

Six percent of patients in pubertal stage $1(4 / 66)$ and $18 \%(41 / 224)$ of patients in pubertal stage 5 $(p<0.03)$ had retinopathy. Duration of diabetes was 
Table 2. Characteristics of the study group

\begin{tabular}{|c|c|c|c|c|}
\hline Retinopathy & $\begin{array}{l}\text { Yes } \\
n=81\end{array}$ & $\begin{array}{l}\text { No } \\
n=476\end{array}$ & $\mathrm{n}$ & $p$ value \\
\hline Sex (female, male) & $47 / 34$ & $232 / 244$ & 557 & 0.1216 \\
\hline Age (years) & $16.8(13.6-20.3)$ & $14.4(12.2-16.8)$ & 557 & 0.0000 \\
\hline Testis volume (ml) & $20(13-25)$ & $15(6.0-25)$ & 230 & 0.1134 \\
\hline Menarche (no/yes) & $17 / 30$ & $106 / 124$ & 277 & 0.21 \\
\hline Age at menarche (years) & $13.0(12.0-13.4)$ & $13.3(13.0-13.5)$ & 277 & 0.0082 \\
\hline Pubertal stage 2 & 7 & 66 & 73 & \\
\hline Pubertal stage 3 & 8 & 51 & 59 & \\
\hline Pubertal stage 4 & 12 & 77 & 89 & \\
\hline Pubertal stage 5 & 42 & 183 & 225 & \\
\hline Pubertal stage not known & 8 & 37 & 45 & \\
\hline
\end{tabular}

Comparison between patients with and without retinopathy using Wilcoxon non-parametrical test, chi-square-test or Fisher's exact test

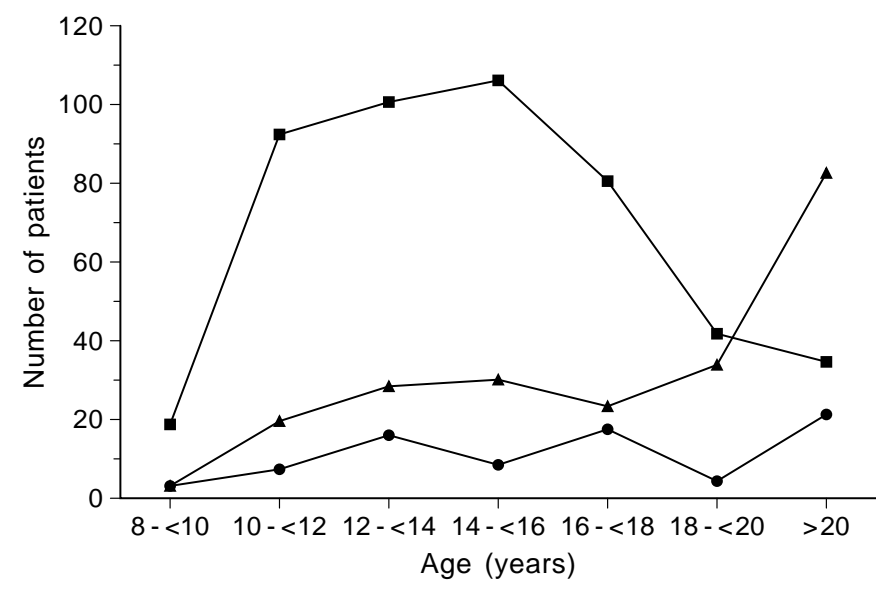

Fig. 1. Age vs retinopathy $\boldsymbol{\Delta}$, patients not examined; $\mathbf{\square}$, patients without signs of retinopathy, $\bullet$, patients with signs of retinopathy

not significantly different in the patients with signs of retinopathy in pubertal stage 1 compared to the postpubertal patients with retinopathy (3.6 [1.5-10.1] years and 8.0 [6.4-9.5] years, respectively, $p<0.10$ ). Life table and Cox proportional hazard analysis showed that neither age nor pubertal development could significantly reduce the survival time when retinopathy occurred ( $p<0.11$ and 0.89 , respectively).

Patients with more advanced stages of retinopathy (40-65) had a longer duration of diabetes (9.0 [8.09.6] years) than those with less advanced stages (2030) (5.4 [3.5-7.8] years, $p<0.03)$ of retinopathy. The youngest patient with level 20 was a 9.5-year-old girl in pubertal stage 1 with a diabetes duration of 1.5 years. Level 40 was demonstrated in 11 patients. Two of these 11 patients had not passed puberty; a 12.8-year-old girl in pubertal stage 3 and an 11.3year-old boy in pubertal stage 2 . Their diabetes durations were respectively 8.8 and 6.9 years and proliferative retinopathy was found in one patient, a 21.5year-old girl with a diabetes duration of 9.5 years.

\section{Discussion}

In this population-based study, we have demonstrated the prevalence of retinopathy to be $14.5 \%$ for any retinopathy and $2.3 \%$ for proliferative and preproliferative retinopathy in children and adolescents.

The dropout rate was $28.6 \%$ of the total sample. There may be a selection bias since the dropouts were significantly older and had longer durations of diabetes. The true prevalence may therefore be higher, since duration and age have invariably been known to be predominant risk factors for retinopathy in children and adolescents with IDDM [2-6].

Only infrequently have studies concerning prevalence of retinopathy in children and adolescents been population based [5]. The majority of studies have been performed on hospital-based populations with a potential risk of selection bias [2-4, 6].

Klein et al. [5] demonstrated in a populationbased study that the prevalence of retinopathy was less than $10 \%$ during the first 5 years after onset of IDDM, in children younger than 13 years and adolescents with onset of diabetes after the age of 13 years. Between 5-10 years after diagnosis of IDDM the prevalence of retinopathy increased to $70 \%$ in the older group, but in the younger group the prevalence was unchanged, suggesting that children are at low risk of retinopathy before the age of 13 and before puberty. Kostraba et al. [14] reported that duration of diabetes before puberty had no impact on later development of retinopathy, but McNally et al. [15] found that surviving without retinopathy was significantly less common among patients with onset of diabetes in the prepubertal period.

None of these groups had examined the pubertal stages, having only approximated it to certain chronological ages. However, it is well-known that pubertal development is dependent on nutritional status, sex, hereditary factors and ethnicity [16]. Murphy et al. [17] and Rogers et al. [18] demonstrated retinopathy 


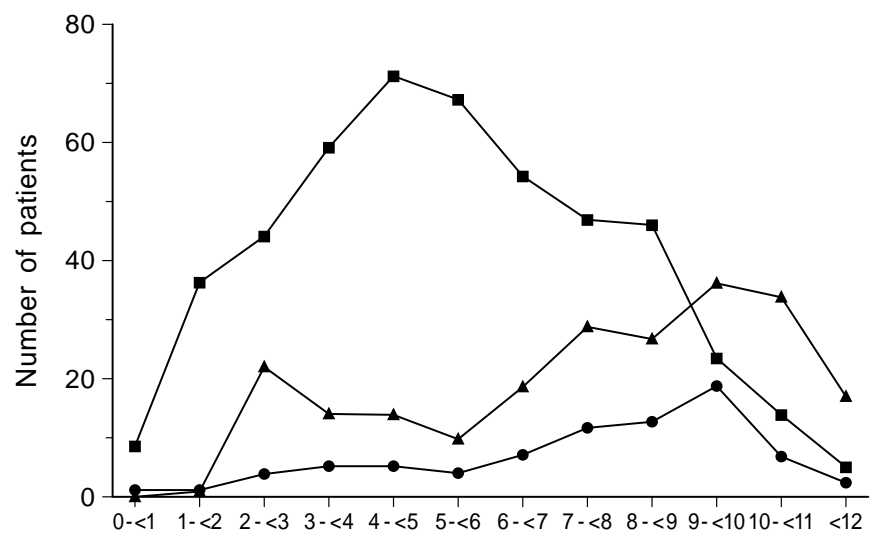

Duration (years)

Fig. 2. Duration vs retinopathy $\boldsymbol{\Delta}$, patients not examined; $\mathbf{\square}$, patients without signs of retinopathy, $\bullet$, patients with signs of retinopathy

in the prepubertal period (stage 1) but the prevalence was much higher in the postpubertal period (stage 5). Murphy et al. [17] demonstrated that the adolescents in Tanner stage 5 had significantly higher mean $\mathrm{HbA}_{1 \mathrm{c}}$. We also found a higher prevalence of retinopathy in pubertal stage 5 than in pubertal stage 1 , but not that pubertal development reduced the survival time without retinopathy.

However, the difference between the prevalence of retinopathy in pubertal stage 5 and in pubertal stage 1 may in reality be even larger because of the proportionally larger number of missing cases in the older age group in our study. Still, it is not clear whether the frequently observed increasing prevalence of retinopathy during puberty is due to increasing duration, pubertal-caused compliance problems or hormonal changes leading to insulin resistance [19].

Even if most of these early retinal changes found in diabetic children do not need photocoagulation therapy, it is important to diagnose retinopathy as soon as possible after the first signs in order to intensify treatment and normalise metabolic control, to prevent and delay further development of retinopathy [20]. Routine fundus photography is a fairly inexpensive method and a simple examination procedure in comparison with the extreme cost, in both economic and psychosocial terms, of missing a few very young diabetic children with retinopathy who may progress to blindness unless detected and treated in time. If a fundus-photograph examination is performed every year from the age of 10 years, instead of examinations every second year from the age of 15 years, it could prevent blindness in at least one patient with the cost-benefit for society of more than 200000 pounds sterling. We therefore strongly advocate screening programmes for retinopathy from the age of 10 years.
A cknowledgements. Financial support was provided by the County of Östergötland, the Sven Jerring Foundation, the Swedish Child Diabetes Foundation, Novo Nordisk Pharma Research Institute, Motala Hospital, the Stig and Ragna Gorthon Foundation and in part by grants from the Swedish Medical Research Council, project No. 12 x-x 734 and project No. 07531. Skilful technical assistance was provided by Ms. I. Franzén, Ms. S. Hellström and Ms. L. Berglert.

\section{References}

1. Retinopathy working party (1991) A protocol for screening for diabetic retinopathy in Europe. Diabet Med 8: 263-267

2. Frank RN, Hoffman WH, Podgor MJ et al. (1980) Retinopathy in juvenile-onset diabetes of short duration. Ophthalmology 87: 1-9

3. Lund-Andersen C, Frost -Larsen K, Starup K (1987) Natural history of diabetic retinopathy in insulin-dependent juvenile diabetes. A longitudinal study. Acta Ophthalmologica 65: 481-486

4. Palmberg P, Smith M, Waltman S et al. (1981) The natural history of retinopathy in insulin-dependent juvenile-onset diabetes. Ophthalmology 88: 613-618

5. Klein R, Klein BE, Moss SE, Davis MD, De Mets DL (1985) Retinopathy in young-onset diabetic patients. Diabetes Care 8: 311-315

6. Burger W, Hövener G, Düsterhus R, Hartmann R, Weber B (1986) Prevalence and development of retinopathy in children and adolescents with type I (insulin-dependent) diabetes mellitus. A longitudinal study. Diabetologia 29: 17-22

7. Dahlquist G, Blom L, Holmgren G, Hägglöf B, Larsson Y, Sterky G, Wall S (1985) The epidemiology of diabetes in Swedish children aged $0-14$ years - a six-year prospective study. Diabetologia 28 : 802-808

8. Nyström L, Dahlquist G, Rewers M,Wall S (1990) The Swedish childhood diabetes study. An analysis of the temporal variation in diabetes incidence 1978-1987. Int J Epidemiol 19: 141-146

9. Fundus Photograph Reading Centre. Department of Ophthalmology, University of Wisconsin Diabetic Retinopathy Study Group (1981) Report 7: A modification of the Airlie House classification of diabetic retinopathy. Invest Ophthalmol Vis Sci 21: 210-266

10. Davis MD, Hubbard LD, Trautman J, Klein R for the KROC collaborative study group (1985) Studies of retinopathy. Methodology for assessment and classification with fundus photographs. Diabetes 34[Suppl 3]:42-49

11. Cohen JA (1960) Coefficient of agreement for nominal scales. Educational and Psychological Measurement 20: 37-46

12. Fleiss JL (1971) Measuring nominal scale agreement among many raters. Psychol Bull 76: 578-382

13. Kleinbaum DG, Kupper LL, Morgenstern H (1982) Epidemiologic research, principle and quantitative methods. Van Nostrand Reinhold, New York

14. Kostraba JN, Dorman JG, Orchard TJ et al. (1989) Contributions of diabetes duration before puberty to development of microvascular complications in IDDM subjects. Diabetes Care 12: 686-693

15. McNally PG, Raymond NT, Swift PG, Hearnshaw JR, Burden AC (1993) Does the prepubertal duration of diabetes influence the onset of microvascular complications? Diabet Med 10: 906-908

16. Prader A (1992) Pubertal growth. Acta Ped Jpn 34: 222-235

17. Murphy RP, Wanda M, Plonick L, Enger C, Vitale S, Patz A (1990) The relationship of puberty to diabetic retinopathy. Arch Ophthalmol 108: 215-218

18. Rogers GD, White B, Shalwitz RA, Palmberg P, Smith ME, Santiago JV (1987) The effect of puberty on the development of early diabetic microvascular disease in insulin-dependent diabetes. Diabet Res Clin Pract 3: 39-44

19. Amiel SA, Sherwin RS, Hintz RL, Gertner JM, Press CM, Tamborlane WV (1984) Effects of diabetes and its control on insulin-like growth factors in young subjects with type I diabetes. Diabetes 33: $1175-1190$

20. Diabetes Control and Complications Trial Research Group (1993). The effect of intensive treatment of diabetes on the development and progression of long term complications in insulin-dependent diabetes mellitus. N Engl J Med 329: 977-986 